\title{
Femtosecond laser microstructuring through optical fibre end faces - inscription of surface gratings and sub-surface splitters
}

\author{
Graham C. B. Lee *a , Kyriacos Kalli ${ }^{+b}$, Charalambos Koutsides ${ }^{b}$, Michael Komodromos ${ }^{\text {c }}$, \\ Kate Sugden ${ }^{\text {a }}$ \\ ${ }^{a}$ Photonics Research Group, Aston University, Birmingham, UK; \\ ${ }^{\mathrm{b}}$ Nanophotonics Research Laboratory, Cyprus University of Technology, 3036, Lemessos, Cyprus; \\ ${ }^{\mathrm{c}}$ Frederick University, Filokyprou, 7 Nicosia 1036, Cyprus
}

\begin{abstract}
We present the results of femtosecond laser microstructuring of optical fibres by direct access of the fibre end face, both at the surface and several hundred microns into the fibre, to realise one- and two-dimensional grating structures and optical fibre splitters, respectively. We show the versatility of this simple but effective inscription method, where we demonstrate classic multiple slit diffraction patterns and show the potential for coarse wavelength division multiplexing for sensor signals. A key advantage for the fibre splitter is that the inscription method avoids the use of oil immersion that compensate for the fibre curvature in the standard side writing method.
\end{abstract}

Keywords: Fourier optics, fibre sensors, fibre end devices, femtosecond laser inscription, diffraction grating, fibre splitter, fibre coupler.

\section{INTRODUCTION}

Femtosecond (fs) lasers can perform high-resolution micromachining and inscription in a wide range of transparent materials, including silica optical fibres. The development of fs lasers has opened up new opportunities in fibre device design and consequently new types of fibre optic sensors [1-4]. It is possible to tune the fs laser parameters such that the nonlinear nature of the light interaction with the material can create either a refractive index change or a void. Most fibre optic sensor devices directly written using fs lasers are machined or inscribed side onto the fibre [5]. A key disadvantage of writing parallel to the fibre axis is the difficult alignment procedure to the core or to a specific point in the cladding due to the curved fibre surface. The most widely adopted method for writing devices in fibre is by applying oil directly onto the fibre so that the curvature of the fibre experienced by the beam is reduced [5]. This method, however, is troublesome without an oil emersion lens as the oil tends to boil and can potentially damage a machining lens. In addition, oil emersion lenses usually have a very short working distance and can be expensive. We present a method of writing Fourier optic devices on the end face of, and waveguide devices in, silica optical fibres using a fs laser without oil. Here we demonstrate several devices that can be fabricated using this method - including a 1D and 2D grating structure written across the fibre end face and a subsurface fibre splitter.

\subsection{Femtosecond laser and setup}

The fs laser writing setup (illustrated in Figure 1), consists of an Amplitude Systemes s-Pulse HP, producing sub $500 f_{s}$ laser pulses at a centre wavelength of $1026 \mathrm{~nm}$, and repetition rate of $100 \mathrm{kHz}$. All inscriptions were conducted using a 100X objective (Mititoyo MPlan Apo NIR Series) with a numerical aperture of 0.5. The FWHM of the laser spot size incident on the fibre was approximately $1.5 \mu \mathrm{m}$. Standard telecoms fibre SMF-28 bare fibre and pigtails were tested for all three presented designs. The fibre adapter or pigtail was placed in a fibre holder with the end face of the fibre positioned perpendicular to the machining lens.

\footnotetext{
*leegcb@aston.ac.uk ; phone +44 121204 3498; fax +44 121204 3682; www.aston.ac.uk

+kkalli@cytanet.com.cy; phone+35725002609; www.cut.ac.cy
} 
The rig with the fibre was mounted on to a sub-nanometre precision XY air-bearing translation stage with a mechanical $\mathrm{Z}$ translation (AerotechABL1000). The machining lens and off axis camera lens were mounted on a second common mechanical $\mathrm{Z}$ translation stage. The motion of the translation stages were precisely controlled by commands written in G-Code.

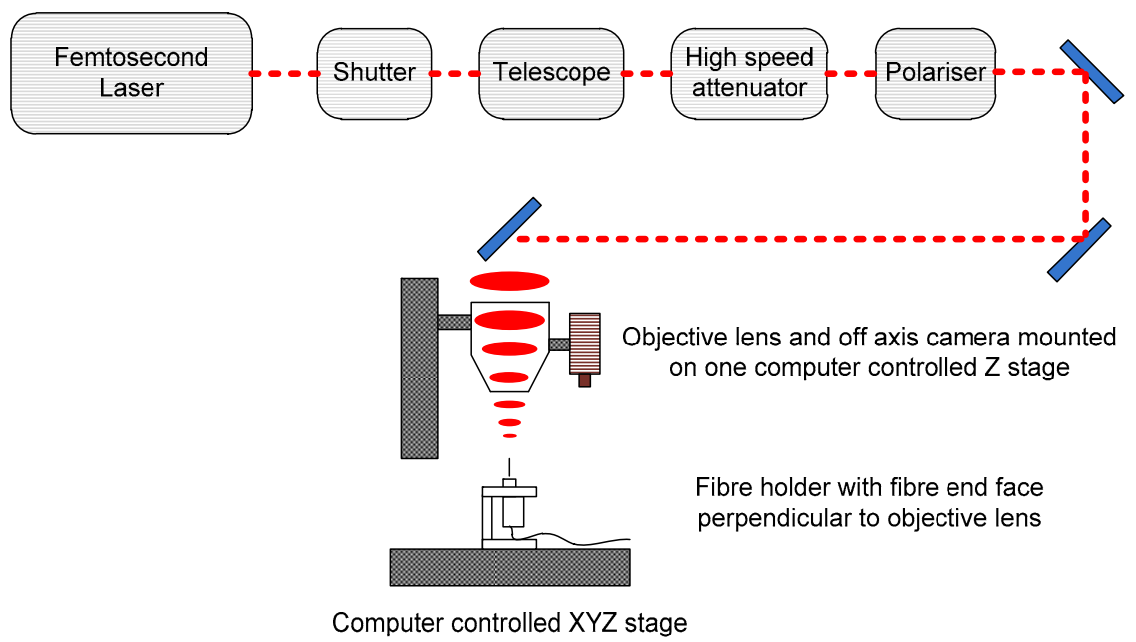

Figure 1. Femtosecond laser setup.

\section{DESIGN}

Three devices have been designed to demonstrate the types of components that can be fabricated for sensing applications, in addition to devices for Fourier optics, using this writing technique. Figure $2 \mathrm{a}$ and $2 \mathrm{~b}$ illustrates the types of structures that can be written on the end of an optical fibre, particular across the core of the fibre. The length of the lines was fixed at $50 \mu \mathrm{m}$ to ensure the core was covered. Devices such as specific diffraction patterns can also be generated under a different pattern design for wavelength division multiplexing (WDM) applications and similarly other devices such as Fresnel lenses can also be fabricated. Figure 2c shows a 3D structure written from and through the end face of the fibre, multiple taps from the core can also be achieved.

a)

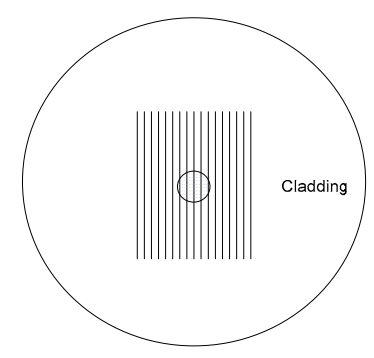

b)

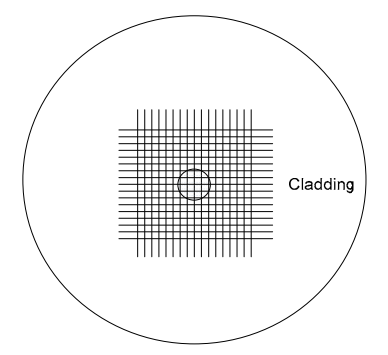

c)

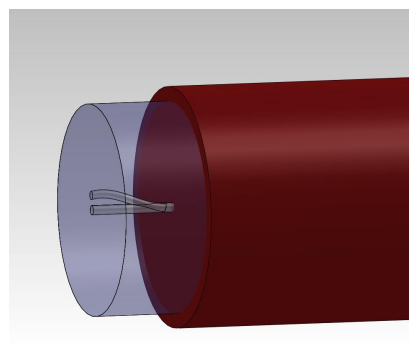

Figure 2. a) 1D grating b) and 2D grating design written across the core of the fibre end. c) Fibre splitter design.

\section{RESULTS AND DISCUSSION}

The grating structure and splitter devices were fabricated in both bare fibre and standard pigtails; results observed from both produced similar or identical results. Several devices were written using each design under a range of laser energies and writing speeds. 


\subsection{Grating structure}

A variety of pitches between the lines in the grating structure were trialled, ranging from 1 to $4 \mu m$. After laser inscription the design pattern on the fibre devices were analysed with an optical profilometer (WYKO 9100NT). In addition, a HeNe laser light source and a supercontinuum white light source (KoherasSuperK Compact, $\lambda$ range from $500-2400 \mathrm{~nm}$ ) were coupled through the fibre device so that the diffraction pattern could be projected on to a flat surface. Figure 3 shows a 1D grating structure device written using $120 \mathrm{~nJ}$ per pulse at a translation speed of $0.1 \mathrm{~mm} / \mathrm{s}$ with a $2 \mu \mathrm{m}$ pitch between the lines. Figure 4 shows a 2D grating structure device written using $120 \mathrm{~nJ}$ per pulse at a translation speed of $0.1 \mathrm{~mm} / \mathrm{s}$ with a $4 \mu \mathrm{m}$ pitch between the lines. Pitches larger than $1.5 \mu \mathrm{m}$ exhibited cleaner and a sharper diffraction pattern. Pitches under this value were likely to have been approaching the fs laser effective spot size; therefore the neighbouring lines began to overlap.

a)

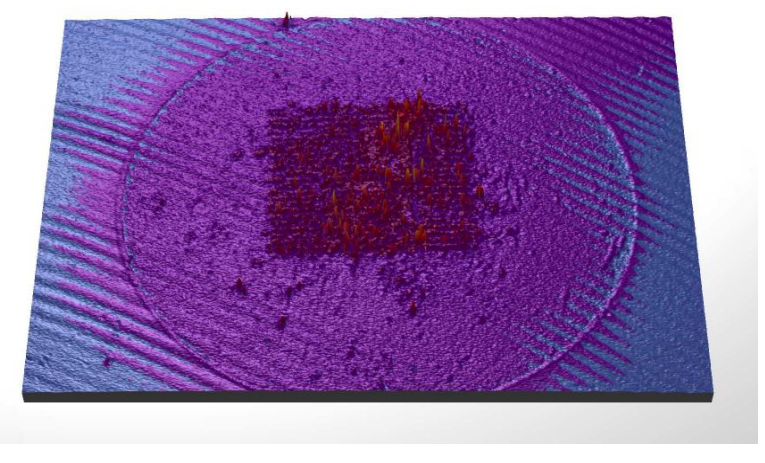

b)

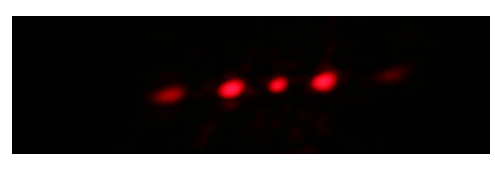

c)

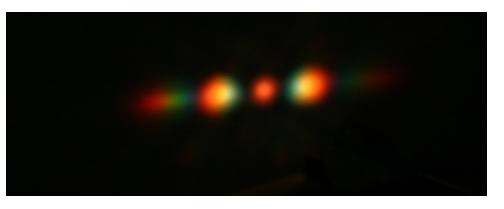

Figure 3. 1D grating structure on the end face of the fibre a) 3D profilometer scan b) diffraction pattern from a HeNe laser light source and the c) diffraction pattern from a fibre-guided supercontinuum white light source.

a)

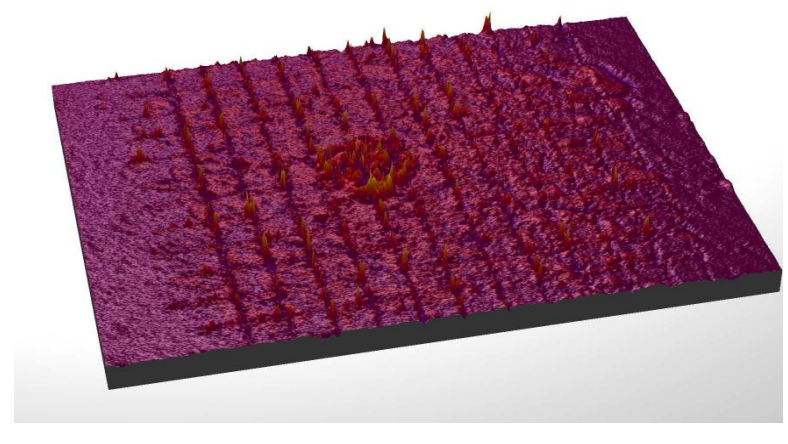

b)

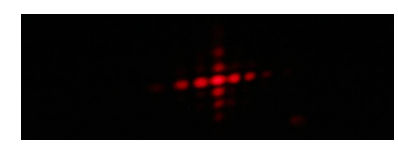

c)

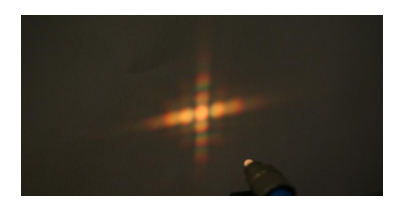

Figure 4. 2D grating structure on the end face of the fibre a) 3D profilometer scan b) diffraction pattern from a HeNe laser light source passing through the device c) diffraction pattern from a fibre-guided supercontinuum white light source.

The results are clearly promising from two perspectives; firstly we observe that the diffraction patterns are of high quality showing excellent symmetry, allowing for increased functionality as a beam splitter, and beam shape controller, and secondly, the light from the supercontinuum source show the possibility for coarse WDM on two axes, allowing for direct wavelength discrimination of light. 


\subsection{Fibre splitter}

The fibre splitter or fibre coupler shows the potential to be able to fabricate waveguide devices with multiple taps from the core to the cladding. With the experimental setup it is possible to control the path from which the light is tapped out of the core to any desired position in the cladding. This includes the capability of varying the angle in which the light is tapped from the core to minimise losses. Figure 5 shows two devices with two different paths from the core to the cladding. Both devices were written with $310 \mathrm{~nJ}$ per pulse at a translation speed of $0.05 \mathrm{~mm} / \mathrm{s}$. The start position of the inscription began at the lowest point in the fibre, the fibre was translated away from the laser focus spot and thus drawing the beam to the surface of the fibre end face. A $50 \mu \mathrm{m}$ line was written very close and parallel to the core to tap the light before being drawn into the cladding.

a)

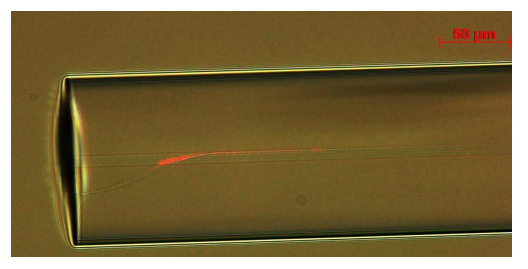

b)

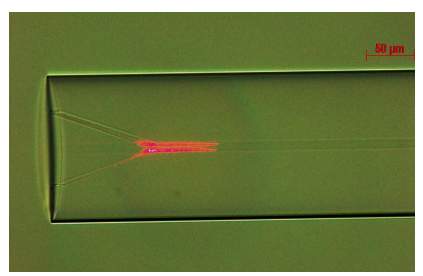

Figure 5. Optical microscope images of a fibre slitter written in SMF-28 with a HeNe light source input, a) one tap from the core, b) two taps from the core.

\section{CONCLUSION}

We have demonstrated the ability to write devices on the end of optical silica fibres, and splitters several hundred microns under the fibre end face using a fs laser. In this paper we have presented several designs written successfully with the fibre end face mounted perpendicular to the machining lens. The potential of the wide variety of devices that can be fabricated with this method for both sensing applications and Fourier optics components were highlighted.

\section{ACKNOWLEDGMENTS}

The authors would like to acknowledge the funding contribution made to this project by COST reference number: COST-STSM-TD1001-9726. G. Lee wishes to thank the Engineering and Physical Sciences Research Council (EPSRC) for funding his Ph.D. K. Kalli and M. Komodromos acknowledge the Research Promotion Foundation, Cyprus, for

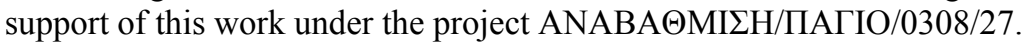

\section{REFERENCES}

[1] K. Kalli, T. Allsop, C. Koutsides et al., "Femtosecond Laser Inscription of Fiber Bragg Gratings with Low Insertion Loss and Minor Polarization Dependence," OSA Technical Digest (CD). BMA3.

[2] R. R. Thomson, H. T. Bookey, N. D. Psaila et al., "Ultrafast-laser inscription of a three dimensional fan-out device for multicore fiber coupling applications," Opt. Express, 15(18), 11691-11697 (2007).

[3] A. Martinez, M. Dubov, I. Khrushchev et al., "Direct writing of fibre Bragg gratings by femtosecond laser," Electronics Letters, 40(19), 1170-1172 (2004).

[4] G. N. Smith, T. Allsop, K. Kalli et al., "Characterisation and performance of a Terfenol-D coated femtosecond laser inscribed optical fibre Bragg sensor with a laser ablated microslot for the detection of static magnetic fields," Opt. Express, 19(1), 363-370 (2011).

[5] Z. Kaiming, D. J. Webb, M. Chengbo et al., "Optical Fiber Cavity Ring Down Measurement of Refractive Index With a Microchannel Drilled by Femtosecond Laser," Photonics Technology Letters, IEEE, 21(22), 1653-1655 (2009). 